\title{
EMISAR: A Dual-frequency, Polarimetric Airborne SAR
}

\author{
Dall, Jørgen; Christensen, Erik Lintz
}

Published in:

Proceedings of the IEEE 2002 International Geoscience and Remote Sensing Symposium

Publication date:

2002

Document Version

Publisher's PDF, also known as Version of record

Link back to DTU Orbit

Citation (APA):

Dall, J., \& Christensen, E. L. (2002). EMISAR: A Dual-frequency, Polarimetric Airborne SAR. In Proceedings of the IEEE 2002 International Geoscience and Remote Sensing Symposium (Vol. 3). IEEE.

\section{General rights}

Copyright and moral rights for the publications made accessible in the public portal are retained by the authors and/or other copyright owners and it is a condition of accessing publications that users recognise and abide by the legal requirements associated with these rights.

- Users may download and print one copy of any publication from the public portal for the purpose of private study or research.

- You may not further distribute the material or use it for any profit-making activity or commercial gain

- You may freely distribute the URL identifying the publication in the public portal

If you believe that this document breaches copyright please contact us providing details, and we will remove access to the work immediately and investigate your claim. 


\title{
EMISAR: A Dual-frequency, Polarimetric Airborne SAR
}

\author{
E.L. Christensen and J. Dall \\ Electromagnetic Systems, Ørsted•DTU, Technical University of Denmark \\ Ørsteds Plads 348, DK-2800 Kgs. Lyngby, Denmark \\ Tel: +45 4525 3800, Fax: +45 4593 1634, E-mail: elc@oersted.dtu.dk
}

\begin{abstract}
EMISAR is a fully polarimetric, dual frequency (L- and Cband) SAR system designed for remote sensing applications. The data are usually processed to $2 \times 2 \mathrm{~m}$ resolution. The system has the capability of C-band cross-track single-pass interferometry and fully polarimetric repeat-pass interferometry. The SAR is operated at high altitudes on a Gulfstream G-3 jet aircraft. The system is very well calibrated and has low sidelobes and low cross-polar contamination. Digital technology has been utilized to realize a flexible and highly stable radar with variable resolution, swath width, and imaging geometry. Thermal control and several calibration loops have been built into the system to ensure system stability and absolute calibration. Accurately measured antenna gains and radiation patterns are included in the calibration. The processing system is developed to support data calibration, which is the key to most of the current applications.
\end{abstract}

\section{INTRODUCTION}

The Danish airborne synthetic aperture radar (EMISAR) was developed for remote-sensing applications with emphasis on system calibration, high resolution, and significant flexibility in acquisition geometry, [1]. EMISAR has been operational as a polarimetric C-band SAR since 1993 and as a dual frequency polarimetric SAR (C- and L-band) since 1995, [2]. Shortly thereafter repeat-pass interferometry was tested and single-pass cross-track interferometry capability was added.

The system has acquired a large amount of high quality data for remote sensing research e.g. ESA's EMAC campaign, [4] and [5], and it has, furthermore, been engaged in a series of calibration experiments and in tests of new features added to the system during the cause of time.

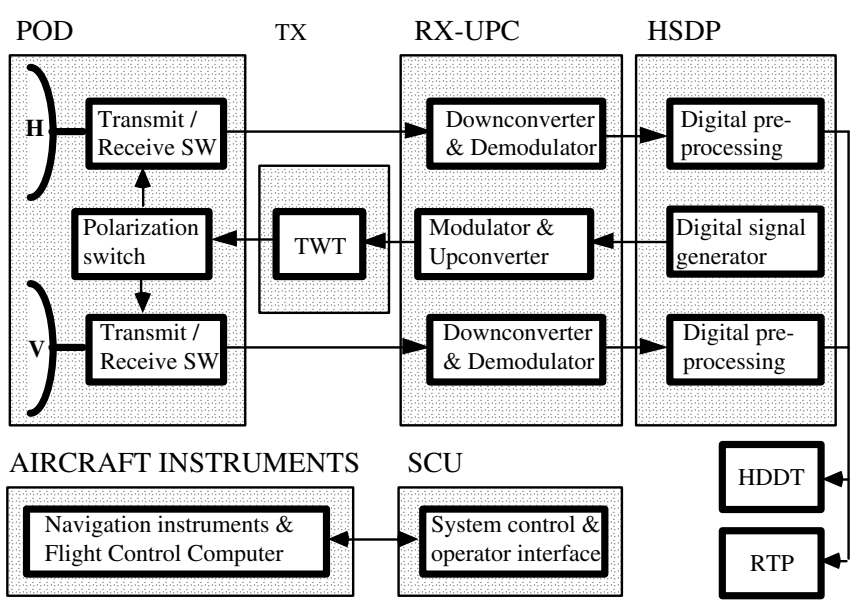

Fig. 1. C-band (or L-band) polarimetric SAR overview diagram. Two separate antennas illustrate the dual polarized antenna.

\section{SYSTEM OVERVIEW}

The system consists of two complete polarimetric SAR systems one for L-band and one for C-band (Fig. 1). Apart from the frequency, the two systems are identical. The two systems share a real-time processor enabling the operator to verify data quality in flight and a $240 \mathrm{Mbit} / \mathrm{s}$ HDDT tape unit for data storage. The SAR control computer navigates the aircraft during data acquisition along the desired flight track based on data from an integrated GPS/INU unit with P-code. The aircraft is navigated by the EMISAR system to within 0.5 $\mathrm{m}$ of the selected flight track, which may deviate from the desired track by up to $10 \mathrm{~m}$ due to GPS offset and drift. Furthermore, GPS information is recorded to enable off-line kinematic GPS processing. This enables very precise flight tracks required for repeat-pass interferometry and high precision geo-location.

EMISAR has two antenna systems. One is a three-axes stabilized antenna in a pod mounted beneath the aircraft. Both an L-band and a C-band antenna can be used but only one at a time. The other system consists of two flush mounted C-band antennas one above the other with a $1.1 \mathrm{~m}$ baseline. All antennas are dual polarized and support the $100 \mathrm{MHz}$ bandwidth of the SAR systems. Together, the two antenna systems support a range of modes, such as simultaneous dual frequency polarimetry or C-band cross track interferometry and L-band polarimetry.

At zero Doppler, the system is usually operated in a mode with $1.5 \times 1.5 \mathrm{~m}$ sample spacing and an unprocessed slant range swath width of $12 \mathrm{~km}$ but the swath width can be increased to $24 \mathrm{~km}$ if a coarser range resolution is accepted. At non-zero Doppler, data are acquired at a finer azimuth resolution and a coarser range resolution.

The system employs an internal calibration system that greatly relieves the dependence on external calibration targets. The system is calibrated via internal signal loops covering the complete system except the antennas just before and after each mapping track. The absolute calibration and the stability of the calibration system have been verified by mapping a Danish calibration site with corner reflectors during each mission.

\section{DATA ACQUISITION MODES}

The sensor supports various signal options, in particular choices of polarizations, interferometric modes, and resolution modes. The proper choice of these options can have significant impact on the applicability of the data for a particular 
purpose and they reflect the necessity of keeping the total data rate (both frequencies) within the capacity of the high density digital tape recorder (HDDT).

The system supports the following polarization modes:

1) Quad, where the transmit polarization is alternately horizontal (H) and vertical (V), and data from all four transmit and receive polarization combinations are stored on tape;

2) Dual, where the transmit polarization is alternated from pulse to pulse, and only the two co-polarized channels are stored on tape; and

3) Single, where only one polarization is transmitted and received.

In addition to this, the flush mounted antennas provide a $\mathrm{C}$ band single pass interferometric capability to allow the generation of high-resolution elevation maps. Although the flush mounted antennas are dual polarized, EMISAR does not support single pass polarimetric interferometry, i.e. acquisition of fully polarimetric data at both ends of the interferometric baseline. The most straightforward implementation would require four instead of two receiver channels at C-band. Alternatively, polarization alternation could be used on the receive side as on the transmit side. However, this would either deteriorate the azimuth ambiguity level or reduce the unambiguous range depending on whether or not the pulse repetition frequency was kept unchanged. Finally, some switches should support pulse-to-pulse switching, i.e. electronic switches should replace the current mechanical switches. More attenuation and hence a poorer signal-to-noise ratio would result.

The sensor can be operated in several resolution modes. The transmitted pulse can be almost any constant amplitude waveform within the constraints of the transmitter duty cycle and the system bandwidth, including linear frequency sweeps (up or down sweeping) with 25, 50, and $100 \mathrm{MHz}$ sweep. In range, optional filtering and subsampling (decimation) can be carried out before the data are stored in the range line buffers, i.e. resolution can be traded for swath width. The range line buffers hold 8192 complex samples. In azimuth, pre-filtering to $1.5 \mathrm{~m}$ sample spacing is normally applied to limit the data rate, but a finer sampling is possible, e.g. $0.75 \mathrm{~m}$ sample spacing if either: 1) only one frequency is recorded; 2) only two polarizations are recorded at each frequency; or 3) the swath is reduced. Normally, data are recorded on tape with $1.5 \mathrm{~m} \times 1.5 \mathrm{~m}$ (range and azimuth) sample spacing, and the unprocessed slant range swath width is $12 \mathrm{~km}$. In wide swath modes the sample spacing is $3.0 \mathrm{~m} \times 1.5 \mathrm{~m}$ or $6.0 \mathrm{~m} \times 1.5 \mathrm{~m}$, and the corresponding swath widths are $24 \mathrm{~km}$ and $48 \mathrm{~km}$.

The full range of different modes, except single-pass interferometry, is supported for both L-band and C-band. Simultaneous operation at both frequencies is also fully supported except for the fact that the present pod can only accommodate one stabilized antenna. Dual frequency data is presently acquired by mounting the L-band antenna in the pod and using one of the flush mounted antennas for C-band polarimetry. However, the present azimuth pre-filters do not support the data from the flush mounted antennas with range varying Doppler centroid. Therefore simultaneous L- and C-band polarimetry necessitates reduced swath operation. If reduced swath is not acceptable the alternative is to change the antenna between two flights. The results stated in this paper for polarimetric performance (e.g. Table 1) are based on the 3-axes stabilized antennas.

\section{POLARIMETRIC DATA PRODUCTS}

The processor is based on the range-Doppler algorithm complemented with motion compensation and calibration algorithms. Three output products are supported:

1) Scattering matrix data in slant range projection

2) Covariance matrix data in pseudo ground range

3) Ground range projected and radiometrically corrected data.

The scattering matrix, $\mathbf{S}$, is a $2 \times 2$ matrix of complex values $\left(\mathrm{S}_{\mathrm{ij}}\right)$, where $\mathrm{j} \in\{\mathrm{H}, \mathrm{V}\}$ is the transmit polarization and $\mathrm{i} \in\{\mathrm{H}, \mathrm{V}\}$ is the receive polarization. The scattering matrix data typically have a sample spacing of $1.5 \times 1.5 \mathrm{~m}$, but they are focused to a nominal resolution of $2.0 \times 2.0 \mathrm{~m}$ in order to suppress sidelobes.

Table 1.

EMISAR C- and L-band performance

\begin{tabular}{|c|c|c|}
\hline System Parameters & & \\
\hline Frequency & C-Band, $5.3 \mathrm{GHz}$ & L-Band $1.25 \mathrm{GHz}$ \\
\hline TWT output power & $2 \mathrm{~kW}$ & $6 \mathrm{~kW}$ \\
\hline Receiver noise figure & $1.5 \mathrm{~dB}$ & $1.5 \mathrm{~dB}$ \\
\hline System loss $(\mathrm{Tx}+\mathrm{Rx}$ side $)$ & $(3.1+0.8)=3.9 \mathrm{~dB}$ & $(3.4+0.9)=4.3 \mathrm{~dB}$ \\
\hline Pulse length & $0.64-20 \mu \mathrm{s}$ & $0.64-20 \mu \mathrm{s}$ \\
\hline Max. bandwidth & $100 \mathrm{MHz}$ & $100 \mathrm{MHz}$ \\
\hline Antenna gain, incl. feeder loss & $26.0 \mathrm{dBi}$ & $17.1 \mathrm{dBi}$ \\
\hline Azimuth $3 \mathrm{~dB}$ beam width & $2.4^{\circ}$ & $10^{\circ}$ \\
\hline Elevation pattern width & $31^{\circ}$ & $42^{\circ}$ \\
\hline Polarization & Fully polarimetric & Fully polarimetric \\
\hline Antenna cross polarization & $<-30 \mathrm{~dB}$ & $<-35 \mathrm{~dB}$ \\
\hline Azimuth ambiguity & $<-30 \mathrm{~dB}$ & $<-30 \mathrm{~dB}$ \\
\hline Resolution in slant range & 2,4 or $8 \mathrm{~m}$ & 2,4 or $8 \mathrm{~m}$ \\
\hline Resolution in azimuth & 2,4 or $8 \mathrm{~m}$ & 2,4 or $8 \mathrm{~m}$ \\
\hline Swath width & 12,24 or $48 \mathrm{~km}$ & 12,24 or $48 \mathrm{~km}$ \\
\hline Flight altitude & Typically $41,000 \mathrm{ft}$ & Typically $41,000 \mathrm{ft}$ \\
\hline Real-time processing & Full resolution & Full resolution \\
\hline $\begin{array}{l}\text { Noise equivalent } \beta_{\mathrm{O}^{\mathrm{N}}} \text {, } \\
\text { distributed target, } 20 \mathrm{~km}\end{array}$ & $-36 \mathrm{~dB}\left(\mathrm{~m}^{2} / \mathrm{m}^{2}\right)$ & $-45 \mathrm{~dB}\left(\mathrm{~m}^{2} / \mathrm{m}^{2}\right)$ \\
\hline PSLR & $-30 \mathrm{~dB}$ & $-25 \mathrm{~dB}$ \\
\hline ISLR & $-28 \mathrm{~dB}$ & $-21 \mathrm{~dB}$ \\
\hline Intrinsic cross-talk terms & $<-30 \mathrm{~dB}$ & $<-35 \mathrm{~dB}$ \\
\hline Calibrated cross-talk terms & $<-35 \mathrm{~dB}$ & $<-35 \mathrm{~dB}$ \\
\hline Equipment in cabin & \multicolumn{2}{|c|}{$600 \mathrm{~kg}$} \\
\hline Pod & \multicolumn{2}{|c|}{$240 \mathrm{~kg}$} \\
\hline $115 \mathrm{~V}, 400 \mathrm{~Hz}$ & \multicolumn{2}{|c|}{$5 \mathrm{~kW}, 6 \mathrm{kVA}$} \\
\hline $28 \mathrm{~V}, \mathrm{DC}$ & \multicolumn{2}{|c|}{$1 \mathrm{~kW}$} \\
\hline Dimensions: Equipment in cabin & \multicolumn{2}{|c|}{3 x 19 " racks, $\mathrm{H}=1.40 \mathrm{~m}$} \\
\hline Pod & \multicolumn{2}{|c|}{$4.59 \times 0.81 \times 0.55 \mathrm{~m}$} \\
\hline
\end{tabular}


The covariance matrix is a $3 \times 3$ matrix as the $\mathrm{HV}$ and $\mathrm{VH}$ channels hold the same information for reciprocal targets. In practice, the average of the two polarimetrically calibrated cross-polarized scattering matrix images is used in order to improve the signal-to-noise ratio. Being a hermitian matrix, the covariance matrix is uniquely defined by the three real elements in the diagonal and the three complex elements above the diagonal. The six different product images are spatially averaged and typically resampled at a $5 \times 5 \mathrm{~m}$ ground pixel spacing using a low pass filter. This filter has a fixed length in the slant range domain in order to obtain a range independent speckle reduction, i.e. the statistics and the equivalent number of looks are constant. The pseudo ground range projection does not take the terrain elevation into account and the conversion of the backscatter coefficient from $\beta_{0}$ to $\sigma_{0}$ does not take the surface slope into account.

The ground range projected and radiometrically corrected data can be produced if an interferometric digital elevation model (DEM) is available, too. The DEM enables a true ground range projection in which the geometric distortion resulting from the topography is eliminated. Also, the DEM enables true line-of-sight vectors to be computed and hence an improved correction for the dual polarized antenna patterns. Finally, from the DEM surface slopes are derived and used to perform a proper conversion of the backscatter coefficient from $\beta_{0}$ to $\sigma_{0}$.

\section{CALIBRATION}

An absolute, polarimetric calibration is applied to EMISAR data. After the absolute radiometric calibration, the radar cross section in square meters divided by $4 \pi$ is found as the energy of a point target, i.e. the sum of the pixel intensities within the mainlobe multiplied by the pixel area. Using the energy rather than the peak intensity implies that, for a homogeneous distributed target, the ensemble average of the pixel intensity (i.e. $\mathrm{I}^{2}+\mathrm{Q}^{2}$ for every pixel) equals the radar brightness $\left(\beta_{0}\right)$ divided by $4 \pi$. The radar brightness in turn equals sigma-naught $\left(\sigma_{0}\right)$ divided by sine of the local incidence angle. The above definition of radiometric calibration is related to the well-known integral method, [6].

The covariance matrix data are calibrated such that the radar cross section of a point target is the sum of the pixel values in the mainlobe times the pixel area and the sigmanaught value of a distributed target is the ensemble average of the pixels. Terrain slope is not taken into account by the sigma-naught calibration.
The internal calibration procedure developed for EMISAR is based on coded pulses, as the measurement signals, generated during calibration time slots just before and after data acquisition, [7]. The calibration comprises channel amplitude- and phase-imbalance correction, absolute radiometric calibration, STC calibration, and noise estimation, and it has a potential for range delay calibration as well.

For the calibration verification and for long-term stability, the internal calibration is complemented by corrections derived from regular external calibration experiments. These corrections are small and very similar from mission to mission and have shown variation ( \pm standard deviation) of e.g. the corrections on absolute calibration of less than $\pm 0.1 \mathrm{~dB}$ at $\mathrm{C}$ band and $\pm 0.5 \mathrm{~dB}$ at L-band.

\section{REFERENCES}

[1] S. Nørvang Madsen, E. Lintz Christensen, N. Skou and J. Dall The Danish SAR System; Design and Initial Tests

IEEE Transactions on Geoscience and Remote Sensing, Vol 29, No 3, May 1991, pp 417-426.

[2] E. Lintz Christensen, N. Skou, J. Dall, Kim W. Woelders, J. Hjelm Jørgensen, J. Granholm, and S. Nørvang Madsen EMISAR: An Absolutely Calibrated Polarimetric L- and C-band SAR IEEE Transactions on Geoscience and Remote Sensing, Vol 36, No 6, November 1998, pp 1852-1865.

[3] S.N. Madsen, N. Skou, J. Granholm, K. Woelders, and E. Lintz Christensen

A System for Airborne SAR Interferometry

AEÜ, Vol. 50, 1996, No. 2, pp 106-111, and Eusar'96, Königswinter, Germany, March 1996, pp 171-176

[4] J. Dall, S. Nørvang Madsen, H. Skriver, S. Savstrup Kristensen, and E. Lintz Christensen

EMISAR Deployment in the EMAC '94 Campaign

ESTEC Contract No. 11095/94/NL/CL, Electromagnetics Institute, The Technical University of Denmark, Lyngby, Denmark, R 600, Sept. 1994.

[5] J. Dall

EMISAR Deployment in the EMAC '95 Campaign

ESTEC Contract No. 11579/95/NL/CN, Electromagnetics Institute, The

Technical University of Denmark, Lyngby, Denmark, R 631, May, 1996.

[6] A.L. Gray, P.W. Vachon, C.E. Livingstone and T.I. Lukowski Synthetic Aperture Radar Calibration Using Reference Reflectors IEEE Transactions on Geoscience and remote Sensing, Vol. 28, No 3, pp. 374-383, May 1990.

[7] J. Dall, N. Skou, E. Lintz Christensen Pulse-based internal calibration of polarimetric SAR

Proceedings of the International Geoscience and Remote Sensing Symposium, IGARSS'94, pp. 2205-2208, Los Angeles, August, 1994. 